\title{
DETECTION OF A RIGID INCLUSION IN AN ELASTIC BODY: UNIQUENESS AND STABILITY
}

\author{
A. Morassi ${ }^{1}$ and E. Rosset ${ }^{2}$ \\ ${ }^{1}$ Università degli Studi di Udine, Dipartimento di Georisorse e Territorio, Udine, Italy, an- \\ tonino.morassi@uniud.it ${ }^{2}$ Universitàdegli Studidi Trieste, Dipartimento di Matematica e In- \\ formatica,Trieste,Italy, rossedi@univ.trieste.it
}

\begin{abstract}
We state uniqueness and stability results for the inverse problem of determining a rigid inclusion inside an isotropic elastic body $\Omega$, from a single measurement of traction and displacement taken on the boundary of $\Omega$.
\end{abstract}

Keywords: inverse problems, linearized elasticity, rigid inclusion.

\section{Introduction}

In this paper we consider the inverse problem of identifying a rigid inclusion inside an elastic body from boundary measurements of traction and displacement. This kind of problems arises, for instance, in non-destructive testing for damage assessment of mechanical specimens, which are possible defective due to the presence of interior rigid inclusions. More precisely, let the elastic body be represented by a bounded domain $\Omega$ in $\mathbf{R}^{2}$, or $\mathbf{R}^{3}$, inside which a possible unknown rigid inclusion $D$ is present. Our aim is to identify $D$ by applying a traction field $\varphi$ at the boundary $\partial \Omega$ and by measuring the induced displacement field on a portion $\Sigma \subset \partial \Omega$.

Working within the framework of the linearized elasticity, where $\mathcal{C}$ denotes the known elasticity tensor of the material, the displacement field $u$ satisfies the following boundary value problem

$$
\left\{\begin{array}{l}
\operatorname{div}(\mathcal{C} \nabla u)=0, \quad \text { in } \Omega \backslash \bar{D}, \\
(\mathcal{C} \nabla u) \nu=\varphi, \quad \text { on } \partial \Omega, \\
u_{\left.\right|_{\partial D} \in \mathcal{R},}
\end{array}\right.
$$

coupled with the global equilibrium condition

$$
\int_{\partial D}(\mathcal{C} \nabla u) \nu \cdot r=0, \quad \text { for every } r \in \mathcal{R}
$$

Please use the following format when citing this chapter:

Morassi, A., and Rosset, E., 2006, in IFIP International Federation for Information Processing, Volume 202, Systems, Control, Modeling and Optimization, eds.

Ceragioli, F., Dontchev, A., Furuta, H., Marti, K., Pandolfi, L., (Boston: Springer), pp. 279-284. 
where $\mathcal{R}$ denotes the linear space of the infinitesimal rigid displacements $r(x)=$ $c+W x$, where $c$ is any constant $n$-vector and $W$ is any constant skew $n \times n$ matrix. We shall assume $\mathcal{C}$ strongly convex and of Lamé type. Problem (1)-(2) admits a solution $u \in H^{1}(\Omega \backslash \bar{D})$, which is unique up to an infinitesimal rigid displacement. In order to specify a unique solution, we shall assume in the sequel the following normalization condition

$$
u=0 \quad \text { on } \partial D .
$$

Therefore, the inverse problem consists in determining the unknown rigid inclusion $D$, appearing in problem (1)-(3), from a single pair of Cauchy data $\{u,(\mathcal{C} \nabla u) \nu\}$ on $\partial \Omega$.

The indeterminacy of the displacement field $u$ and the consequent arbitrariness of the normalization (3) which we have chosen, lead to the following formulation of the uniqueness and stability issues.

Uniqueness issue.

Given two solutions $u_{i}$ to (1)-(3) when $D=D_{i}, i=1,2$, satisfying

$$
\begin{gathered}
\left(\mathcal{C} \nabla u_{i}\right) \nu=\varphi, \quad \text { on } \partial \Omega, \\
u_{1}-u_{\left.2\right|_{\Sigma}} \in \mathcal{R},
\end{gathered}
$$

does $D_{1}=D_{2}$ hold?

Stability issue.

Given two solutions $u_{i}$ to (1)-(3) when $D=D_{i}, i=1,2$, satisfying

$$
\begin{gathered}
\left(\mathcal{C} \nabla u_{i}\right) \nu=\varphi, \quad \text { on } \partial \Omega, \\
\min _{r \in \mathcal{R}}\left\|\left(u_{1}-u_{2}\right)-r\right\|_{L^{2}(\Sigma)}<\epsilon, \quad \text { for some } \epsilon>0,
\end{gathered}
$$

to evaluate the rate at which the Hausdorff distance between $D_{1}$ and $D_{2}$ tends to zero as $\epsilon$ tends to zero.

In this note we present the uniqueness and stability results obtained in [4], to which we refer for the proofs.

In particular, we have obtained uniqueness under the assumption that $\partial D$ is of $C^{1}$ class, see Theorem 1 . The proof of uniqueness is mainly based on the weak unique continuation principle for solutions to the Lamé system (first established by Weck [5]), the uniqueness for the corresponding Cauchy problem, (see, for instance, [2] and [3]), and geometrical arguments related to the structure of the linear space $\mathcal{R}$ which involve different techniques according to the space dimension.

This inverse problem is severely ill-posed from the point of view of stability and, therefore, only a weak rate of convergence, under some a priori information 
on the unknown boundary $\partial D$, can be expected. In [4] a constructive stability estimate of $\log$ - $\log$ type, assuming $C^{1, \alpha}$ regularity of $\partial D, 0<\alpha \leq 1$, has been proved, see Theorem 2 for a precise statement.

The key ingredients to prove stability are quantitative versions of the unique continuation principle, precisely: a three spheres inequality for solutions to the Lamé system, which was obtained in [1]; stability estimates for solutions to the Cauchy problem, obtained in [3]; a stability estimate of continuation from the interior for a mixed boundary value problem obtained in [4]. The complications of geometrical character arising in the proof of uniqueness, due to the general form of the condition (7), become significantly harder in the stability context, see the geometrical Lemma 7.1 in [4].

\section{A priori information}

In the sequel we use the following notation.

Let $\Omega$ be a bounded domain in $\mathbf{R}^{n}$. Given $k$, $\alpha$, with $k \in \mathbf{N}, 0<\alpha \leq 1$, we say that a portion $S$ of $\partial \Omega$ is of class $C^{k, \alpha}$ with constants $\rho_{0}, M_{0}>0$, if, for any $P \in S$, there exists a rigid transformation of coordinates under which we have $P=0$ and

$$
\Omega \cap B_{\rho_{0}}(0)=\left\{x=\left(x^{\prime}, x_{n}\right) \in B_{\rho_{0}}(0) \quad \mid \quad x_{n}>\psi\left(x^{\prime}\right)\right\},
$$

where $x^{\prime}=\left(x_{1}^{\prime}, \ldots, x_{n-1}^{\prime}\right) \in \mathbf{R}^{n-1}$ and $\psi$ is a $C^{k, \alpha}$ function on $B_{p_{0}}^{\prime}(0)=$ $B_{\rho_{0}}(0) \cap\left\{x_{n}=0\right\} \subset \mathbf{R}^{n-1}$ satisfying

$$
\begin{gathered}
\psi(0)=0, \\
\nabla \psi(0)=0, \quad \text { when } k \geq 1, \\
\|\psi\|_{C^{k, \alpha}\left(B_{\rho_{0}}^{\prime}(0)\right)} \leq M_{0} .
\end{gathered}
$$

When $k=0, \alpha=1$, we also say that $S$ is of Lipschitz class with constants $\rho_{0}$, $M_{0}$.

We denote by $\mathbf{M}^{m \times n}$ the space of $m \times n$ real valued matrices and by $\mathcal{L}(X, Y)$ the space of bounded linear operators between Banach spaces $X$ and $Y$. When $m=n$, we shall also denote $\mathbf{M}^{n}=\mathbf{M}^{n \times n}$.

For every $n \times n$ matrix $A$ and for every $\mathcal{C} \in \mathcal{L}\left(\mathbf{M}^{n}, \mathbf{M}^{n}\right)$, we use the following notation:

$$
(\mathcal{C} A)_{i j}=\sum_{k, l=1}^{n} \mathcal{C}_{i j k l} A_{k l}
$$

Let us introduce the linear space of the infinitesimal rigid displacements

$$
\mathcal{R}=\left\{r(x)=c+W x, c \in \mathbf{R}^{n}, W \in \mathbf{M}^{n}, W+W^{T}=0\right\},
$$


where $x$ is the vector position of a generic point in $\mathbf{R}^{n}$.

Let us list here the a priori information needed for stability.

i) A priori information on the domain.

We assume that $\Omega$ is a bounded domain in $\mathbf{R}^{n}$ such that

$$
|\Omega| \leq M_{1},
$$

for some $M_{1}>0$, where $|\Omega|$ denotes the Lebesgue measure of $\Omega$.

For the sake of simplicity, we assume in the sequel that

$$
\partial \Omega \text { is connected, }
$$

but we emphasize that all the results stated in the next Section continue to hold in the general case when (11) is removed.

We assume that $\Omega$ contains an open, connected, rigid inclusion $D$ such that

$$
\Omega \backslash \bar{D} \text { is connected, }
$$

$$
\partial D \text { is connected, }
$$

and

$$
\operatorname{dist}(D, \partial \Omega) \geq \rho_{0} .
$$

Moreover, we assume that we can select an open portion $\Sigma$ within $\partial \Omega$ (representing the portion of the boundary where measurements are taken) such that for some $P_{0} \in \Sigma$

$$
\partial \Omega \cap B_{\rho_{0}}\left(P_{0}\right) \subset \Sigma .
$$

Regarding the regularity of the boundaries, given $\alpha, M_{0}, 0<\alpha \leq 1, M_{0}>0$, we assume that

$$
\begin{aligned}
& \partial \Omega \text { is of class } C^{1, \alpha} \text { with constants } \rho_{0}, M_{0}, \\
& \partial D \text { is of class } C^{1, \alpha} \text { with constants } \rho_{0}, M_{0},
\end{aligned}
$$

and, moreover, that

$$
\Sigma \text { is of class } C^{2, \alpha} \text { with constants } \rho_{0}, M_{0} .
$$

ii) Assumptions about the boundary data.

On the Neumann data $\varphi$ appearing in problem (1) we assume that

$$
\varphi \in H^{-\frac{1}{2}}\left(\partial \Omega, \mathbf{R}^{n}\right), \quad \varphi \not \equiv 0,
$$


the compatibility condition

$$
\int_{\partial \Omega} \varphi \cdot r=0, \quad \text { for every } r \in \mathcal{R}
$$

and that, for a given constant $F>0$,

$$
\frac{\|\varphi\|_{H^{-\frac{1}{2}}\left(\partial \Omega, \mathbf{R}^{n}\right)}}{\|\varphi\|_{H^{-1}}\left(\partial \Omega, \mathbf{R}^{n}\right)} \leq F .
$$

iii) Assumptions about the elasticity tensor.

We assume that the elastic material is isotropic, that is the elasticity tensor field $\mathcal{C}=\mathcal{C}(x) \in \mathcal{L}\left(\mathbf{M}^{n}, \mathbf{M}^{n}\right)$ has components $\mathcal{C}_{i j k l}$ given by

$$
\mathcal{C}_{i j k l}(x)=\lambda(x) \delta_{i j} \delta_{k l}+\mu(x)\left(\delta_{k i} \delta_{l j}+\delta_{l i} \delta_{k j}\right), \quad \text { for every } x \in \bar{\Omega},
$$

where $\lambda=\lambda(x)$ and $\mu=\mu(x)$ are the Lamé moduli. Moreover we assume that the Lamé moduli satisfy the $C^{1,1}$ regularity condition

$$
\|\mu\|_{C^{1,1}(\bar{\Omega})}+\|\lambda\|_{C^{1,1}(\bar{\Omega})} \leq M
$$

and the strong convexity condition

$$
\mu(x) \geq \alpha_{0}, \quad 2 \mu(x)+n \lambda(x) \geq \beta_{0}, \quad \text { for every } x \in \bar{\Omega},
$$

where $M, \alpha_{0}, \beta_{0}$ are given positive constants.

We shall refer to the set of constants $\alpha, \rho_{0}, M_{0}, M_{1}, F, \alpha_{0}, \beta_{0}, M$ as to the a priori data.

By standard variational arguments it is easy to see that problem (1)-(3) admits a unique solution $u \in H^{1}\left(\Omega \backslash \bar{D}, \mathbf{R}^{n}\right)$ such that

$$
\|u\|_{H^{1}\left(\Omega \backslash \bar{D}, \mathbf{R}^{n}\right)} \leq C\|\varphi\|_{H^{-\frac{1}{2}}\left(\partial \Omega, \mathbf{R}^{n}\right)}
$$

where $C>0$ only depends on $\alpha_{0}, \beta_{0}, \rho_{0}, M_{0}$ and $M_{1}$.

\section{Main results}

\section{Theorem 1 (Uniqueness)}

Let $\Omega$ be a bounded domain satisfying (11) and having Lipschitz boundary. Let $D_{i}, i=1,2$, be two domains compactly contained in $\Omega$, having $C^{1}$ boundary and satisfying (12) and (13). Moreover, let $\Sigma$ be an open portion of $\partial \Omega$ of class $C^{2, \alpha}$. Let $u_{i} \in H^{1}\left(\Omega \backslash \overline{D_{i}}, \mathbf{R}^{n}\right)$ be the solution to (1)-(3), when $D=D_{i}$, $i=1,2$, let (19), (20) be satisfied and let the elasticity tensor $\mathcal{C}$ of Lamé type, with Lamé moduli $\lambda$ and $\mu$ of $C^{1,1}$ class satisfying $\mu>0,2 \mu+n \lambda>0$ in $\bar{\Omega}$. If we have

$$
\left(u_{1}-u_{2}\right)_{\left.\right|_{\Sigma}} \in \mathcal{R}
$$


then

$$
D_{1}=D_{2}
$$

\section{Theorem 2 (Stability)}

Let $\Omega$ be a domain satisfying (10), (11) and (16). Let $D_{i}, i=1,2$, be two connected open subsets of $\Omega$ satisfying (12), (13), (14) and (17). Moreover, let $\Sigma$ be an open portion of $\partial \Omega$ satisfying (15) and (18). Let $u_{i} \in H^{1}\left(\Omega \backslash \overline{D_{i}}, \mathbf{R}^{n}\right)$ be the solution to (1)-(3), when $D=D_{i}, i=1,2$, and let (19)-(24) be satisfied. If, given $\epsilon>0$, we have

$$
\left\|u_{1}-u_{2}-\bar{r}\right\|_{L^{2}\left(\Sigma, \mathbf{R}^{n}\right)}=\min _{r \in \mathcal{R}}\left\|u_{1}-u_{2}-r\right\|_{L^{2}\left(\Sigma, \mathbf{R}^{n}\right)} \leq \epsilon
$$

then we have

$$
d_{\mathcal{H}}\left(\partial D_{1}, \partial D_{2}\right) \leq \omega\left(\frac{\epsilon}{\|\varphi\|_{H^{-\frac{1}{2}}\left(\partial \Omega, \mathbf{R}^{n}\right)}}\right)
$$

and

$$
d_{\mathcal{H}}\left(\overline{D_{1}}, \overline{D_{2}}\right) \leq \omega\left(\frac{\epsilon}{\|\varphi\|_{H^{-\frac{1}{2}}\left(\partial \Omega, \mathbf{R}^{n}\right)}}\right),
$$

where $\omega$ is an increasing continuous function on $[0, \infty)$ which satisfies

$$
\omega(t) \leq C(\log |\log t|)^{-\eta}, \quad \text { for every } t, 0<t<e^{-1},
$$

and $C, \eta, C>0,0<\eta \leq 1$, are constants only depending on the a priori data.

Here $d_{\mathcal{H}}$ denotes the Hausdorff distance between bounded closed sets of $\mathbf{R}^{n}$.

\section{References}

[1] G. Alessandrini, A. Morassi. Strong unique continuation for the Lamé system of elasticity. Comm. Partial Differential Equations 26:1787-1810, 2001.

[2] M. Eller, V. Isakov, G. Nakamura, D. Tataru. Uniqueness and stability in the Cauchy problem for Maxwell and elasticity systems. In Nonlinear Partial Differential Equations, Vol. 16, College de France Seminar. Chapman and Hill/CRC, 2000.

[3] A. Morassi, E. Rosset. Stable determination of cavities in elastic bodies. Inverse Problems 20:453-480, 2004.

[4] A. Morassi, E. Rosset. Uniqueness and stability in determining a rigid inclusion in an elastic body. Submitted, 2005.

[5] N. Weck. Außenraumaufgaben in der Theorie stationärer Schwingungen inhomogener elasticher Körper. Math. Z. 111:387-398, 1969. 